\title{
Acquired Testicular Failure
}

National Cancer Institute

\section{Source}

National Cancer Institute. Acquired Testicular Failure. NCI Thesaurus. Code C131091.

Testicular failure, the cause of which is not present at birth. 